\title{
MMSE Beamforming for SC-FDMA Transmission over MIMO ISI Channels
}

\author{
Uyen Ly Dang, ${ }^{1}$ Michael A. Ruder, ${ }^{1}$ Robert Schober, ${ }^{2}$ and Wolfgang H. Gerstacker ${ }^{1}$ \\ ${ }^{1}$ Institute of Mobile Communications, University of Erlangen-Nürnberg, Cauerstraße 7, 91058 Erlangen, Germany \\ ${ }^{2}$ Department of Electrical and Computer Engineering, University of British Columbia, Vancouver, BC, Canada V6T1Z4
}

Correspondence should be addressed to Wolfgang H. Gerstacker, gersta@lnt.de

Received 12 May 2010; Revised 14 October 2010; Accepted 9 November 2010

Academic Editor: D. D. Falconer

Copyright (c) 2011 Uyen Ly Dang et al. This is an open access article distributed under the Creative Commons Attribution License, which permits unrestricted use, distribution, and reproduction in any medium, provided the original work is properly cited.

\begin{abstract}
We consider transmit beamforming for single-carrier frequency-division multiple access (SC-FDMA) transmission over frequency-selective multiple-input multiple-output (MIMO) channels. The beamforming filters are optimized for minimization of the sum of the mean-squared errors (MSEs) of the transmitted data streams after MIMO minimum mean-squared error linear equalization (MMSE-LE), and for minimization of the product of the MSEs after MIMO MMSE decision-feedback equalization (MMSE-DFE), respectively. We prove that for SC-FDMA transmission in both cases eigenbeamforming, diagonalizing the overall channel, together with a nonuniform power distribution is the optimum beamforming strategy. The optimum power allocation derived for MMSE-LE is similar in spirit to classical results for the optimum continuous-time transmit filter for linear modulation formats obtained by Berger/Tufts and Yang/Roy, whereas for MMSE-DFE the capacity achieving waterfilling strategy well known from conventional single-carrier transmission schemes is obtained. Moreover, we present a modification of the beamformer design to mitigate an increase of the peak-to-average power ratio (PAPR) which is in general associated with beamforming. Simulation results demonstrate the high performance of the proposed beamforming algorithms.
\end{abstract}

\section{Introduction}

Single-carrier frequency-division multiple access (SCFDMA) transmission, also referred to as discrete Fourier transform (DFT) spread orthogonal frequency-division multiple access (OFDMA), has been selected for the uplink of the E-UTRA Long-Term Evolution (LTE) mobile communications system [1]. In comparison to standard OFDMA, SCFDMA enjoys a reduced peak-to-average power ratio (PAPR) enabling a low-complexity implementation of the mobile terminal [2]. SC-FDMA is employed along with multipleinput multiple-output (MIMO) techniques in LTE in order to further improve coverage and capacity. Another advantage of SC-FDMA is that relatively simple frequency-domain minimum mean-squared error linear equalization (MMSELE) techniques $[3,4]$ can be applied for signal recovery at the base station, if a frequency-selective MIMO channel is present and introduces intersymbol interference (ISI). Incorporating additional MMSE noise (error) prediction, tailored for single-carrier transmission techniques with cyclic convolution, compare with for example, [5], an MMSE decision-feedback equalization (MMSE-DFE) structure results with enhanced performance compared to MMSE-LE.

In order to fully exploit the potential benefits of MIMO transmission, closed-loop transmit beamforming should be employed, compare with for example, $[6,7]$, where a pragmatic eigenbeamforming algorithm using unitary precoding matrices in conjunction with uniform power allocation across all subcarriers has been introduced for SC-FDMA MIMO transmission with MMSE-LE. However, in this work, we show that eigenbeamforming with uniform power allocation is suboptimum. We prove that beamforming filters, minimizing the mean-squared error (MSE) after MMSE-LE, lead to eigenbeamforming with a nonuniform power allocation across the subcarriers. The optimum power allocation policy is derived and shown to be similar in spirit to classical results for the optimum continuous-time transmit filters for a conventional single-carrier transmission, compare with [8], that is, it is given by an inverse waterfilling scheme. For MMSE-DFE, it is shown that also eigenbeamforming 
together with a nonuniform power allocation across the subcarriers is optimal in general. Here the optimum power allocation policy is proved to be given by classical capacity achieving waterfilling, again similar to conventional singlecarrier transmission, compare with [9].

Simulation results demonstrate the high performance of the proposed beamforming schemes and show that beamforming introduces a certain increase in the peak-to-average power ratio (PAPR). For PAPR reduction, symbol amplitude clipping has been proposed in [7], which is known to introduce in-band signal distortion. Therefore, in this work, a modified version of the selected mapping (SLM) method $[10,11]$ is used, which can be incorporated without loss of optimality into the beamformer design to keep the increase of the PAPR at a minimum.

This paper is organized as follows. In Section 2, the underlying system model for a single-user MIMO SC-FDMA transmission is described. MMSE-LE and MMSE-DFE for MIMO SC-FDMA transmission are introduced in Sections 3 and 4, respectively. MMSE beamforming for MMSE-LE and MMSE-DFE are derived in Sections 5 and 6, respectively, and a method for PAPR reduction is proposed in Section 7. Numerical results for beamforming and the proposed PAPR reduction method are presented in Section 8 , and some conclusions and suggestions for future work are provided in Section 9.

Notation 1. $\mathscr{E}\{\cdot\},(\cdot)^{T}$, and $(\cdot)^{H}$ denote expectation, transposition, and Hermitian transposition, respectively. Bold lowercase letters and bold uppercase letters stand for column vectors and matrices, respectively. An exception are frequency-domain vectors for which also bold upper case letters are used. $[\mathbf{A}]_{m, n}$ denotes the element in the $m$ th row and $n$th column of matrix $\mathbf{A} ; \mathbf{I}_{X}$ is the $X \times X$ identity matrix, $\mathbf{0}_{X \times Y}$ stands for an $X \times Y$ all-zero matrix, and $\operatorname{diag}\left\{x_{1}, x_{2}, \ldots, x_{n}\right\}$ is a diagonal matrix with elements $x_{1}, x_{2}, \ldots, x_{n}$ on the main diagonal. $\operatorname{tr}(\cdot)$ and $\operatorname{det}(\cdot)$ refer to the trace and determinant of a matrix, respectively. $\mathbf{W}_{X}$ denotes the unitary $X$-point DFT matrix and $\otimes$ denotes cyclic convolution.

\section{System Model}

We consider single-user SC-FDMA transmission over a frequency-selective MIMO channel. Here, we assume $N_{t}=2$ transmit antennas, which is the most realistic setting for the LTE uplink, and $N_{r} \geq 2$ receive antennas. The derived solution can be generalized in a straightforward way to any number of transmit antennas $N_{t}>2$.

Figure 1 shows the considered SC-FDMA transmitter. After channel encoding of binary symbols and interleaving, Gray mapping to a quadrature amplitude modulation (QAM) signal constellation is applied. The corresponding symbols of both transmit branches $a_{i}[k], i \in\{1,2\}$, $k \in\{0,1, \ldots, M-1\}$ of variance $\sigma_{a}^{2}=\mathcal{E}\left\{\left|a_{i}[k]\right|^{2}\right\}$ are independent and identically distributed (i.i.d.), where $M$ symbols form one block. An $M$-point DFT is applied to each block $\mathbf{a}_{i} \triangleq\left[a_{i}[0] a_{i}[1] \cdots a_{i}[M-1]\right]^{T}$ leading to vector $\mathbf{A}_{i} \triangleq \mathbf{W}_{M} \mathbf{a}_{i}$ in the frequency domain with

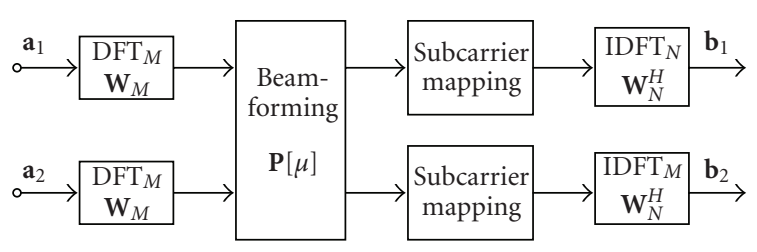

FIGURE 1: Transmitter with SC-FDMA signal processing and beamforming.

$\mathbf{A}_{i}=\left[A_{i}[0] A_{i}[1] \cdots A_{i}[M-1]\right]^{T}$. Subsequently, the frequency domain symbols are mapped onto $N$ subcarriers, resulting in frequency domain vectors $\mathbf{B}_{i}$ of size $N$. Hereby, mapping is done by the assignment to $M$ consecutive subcarriers beginning from the $\nu_{0}$ th subcarrier, which can be represented as

$$
\mathbf{B}_{i}=\mathbf{K} \mathbf{A}_{i}
$$

with the assignment matrix

$$
\mathbf{K} \triangleq\left[\mathbf{0}_{\nu_{0} \times M}^{T} \mathbf{I}_{M}^{T} \mathbf{0}_{\left(N-M-\nu_{0}\right) \times M}^{T}\right]^{T} .
$$

Using an $N$-point inverse (I)DFT, time-domain transmit vectors $\mathbf{b}_{i}$ with elements $b_{i}[\kappa], \kappa \in\{0,1, \ldots, N-1\}$, are computed, that is, $\mathbf{b}_{i} \triangleq \mathbf{W}_{N}^{H} \mathbf{B}_{i}$.

If additional beamforming is employed at the transmitter side, a cyclic $2 \times 2$ matrix filter is applied to input vector $\left[b_{1}[\kappa] b_{2}[\kappa]\right]^{T}$ in each time step. This can be implemented also in the $M$-point DFT domain by forming sequences $\tilde{A}_{1}[\mu]$ and $\tilde{A}_{2}[\mu]$ via

$$
\left[\tilde{A}_{1}[\mu] \tilde{A}_{2}[\mu]\right]^{T}=\mathbf{P}[\mu]\left[A_{1}[\mu] A_{2}[\mu]\right]^{T}
$$

with a $2 \times 2$ beamformer frequency response matrix $\mathbf{P}[\mu]$ as shown in Figure 1. For the subcarrier assignment, sequences $\widetilde{A}_{i}[\mu]$ instead of $A_{i}[\mu]$ are used in (1), that is, $\mathbf{A}_{i}$ is replaced by a vector $\widetilde{\mathbf{A}}_{i}$ constructed from sequence $\tilde{A}_{i}[\mu]$.

A cyclic prefix of length $L_{c}$ is added to vectors $\mathbf{b}_{i}$ and the sequences $b_{i, c}[\kappa]$ corresponding to $\mathbf{b}_{i, c} \triangleq\left[b_{i}\left[N-L_{c}\right] b_{i}[N-\right.$ $\left.\left.L_{c}+1\right] \cdots b_{i}[N-1] \mathbf{b}_{i}^{T}\right]^{T}$, that is, $b_{i, c}[0]=b_{i}\left[N-L_{c}\right]$, $b_{i, c}[1]=b_{i}\left[N-L_{c}+1\right], \ldots, b_{i, c}\left[N+L_{c}-1\right]=b_{i}[N-1]$, form an SC-FDMA transmit symbol. (Here, index " $c$ " stands for the additional cyclic prefix.) The signal at the $l$ th receive antenna, $l \in\left\{1,2, \ldots, N_{r}\right\}$, is

$$
r_{l, c}[k]=\sum_{i=1}^{2} \sum_{\lambda=0}^{L-1} h_{l, i}[\lambda] b_{i, c}[\kappa-\lambda]+n_{l}[\kappa],
$$

where the discrete-time subchannel impulse response $h_{l, i}[\lambda]$ of length $L$ characterizes transmission from the $i$ th transmit antenna to the $l$ th receive antenna including transmit and receiver input filtering. (Symbols from the preceding SCFDMA symbol can be ignored in the model because they do not contribute after removal of the cyclic prefix.) During the transmission of each slot consisting of several vectors (SC-FDMA symbols) $\mathbf{b}_{i, c}$, the MIMO channel is assumed 
to be constant but it may change randomly from slot to slot. $n_{l}[\kappa]$ denotes spatially and temporally white Gaussian noise of variance $\sigma_{n}^{2}$. In the receiver, the cyclic prefix is first removed, eliminating interference between adjacent SCFDMA symbols if $L_{c} \geq L-1$, and after an $N$-point DFT the received frequency domain vector $\mathbf{R}_{l}$ at antenna $l$ can be represented as

$$
\mathbf{R}_{l}=\sum_{i=1}^{2} \mathbf{H}_{l, i} \mathbf{B}_{i}+\mathbf{N}_{l},
$$

corresponding to a cyclic convolution in the time domain, where $\mathbf{H}_{l, i}=\operatorname{diag}\left\{H_{l, i}[0], H_{l, i}[1], \ldots, H_{l, i}[N-1]\right\}$ with $H_{l, i}[\nu] \triangleq \sum_{\lambda=0}^{L-1} h_{l, i}[\lambda] \mathrm{e}^{-j(2 \pi / N) \nu \lambda}$, and $\mathbf{N}_{l}$ is the frequencydomain noise vector.

\section{MMSE-LE for SC-FDMA}

MMSE-LE for a MIMO SC-FDMA transmission has been outlined for example, in $[4,6]$. The optimum filtering matrix for joint processing of vectors $\mathbf{R}_{l}$ is given by $[4,(8)]$, delivering estimates $y_{i}[k], i \in\{1,2\}$, with $y_{i}[k]=a_{i}[k]+$ $e_{i}[k]$, where the error sequences $e_{i}[k]$ have variances $\sigma_{e, i}^{2}$, $i \in\{1,2\}$. Essentially, MMSE equalization can be realized by frequency-domain MIMO MMSE filtering with matrix

$$
\mathbf{F}[\mu]=\left(\mathbf{H}^{H}[\mu] \mathbf{H}[\mu]+\zeta \mathbf{I}_{2}\right)^{-1} \mathbf{H}^{H}[\mu],
$$

where $[\mathbf{H}[\mu]]_{l, i} \triangleq H_{l, i}\left[\nu_{0}+\mu\right]$ and $\zeta \triangleq \sigma_{n}^{2} / \sigma_{a}^{2}$, applied independently to each relevant frequency component $\mu$, and subsequent IDFT operations, compare with for example, $[4,6]$. For beamforming filter design, the covariance matrix of the error vector $\mathbf{e}[k] \triangleq\left[e_{1}[k] e_{2}[k]\right]^{T}, \Phi_{e e} \triangleq$ $\mathscr{E}\left\{\mathbf{e}[k] \mathbf{e}^{H}[k]\right\}$, is needed and calculated in the following. Defining the equalizer output vector $\mathbf{y}[k] \triangleq\left[y_{1}[k] y_{2}[k]\right]^{T}$ and using the above-mentioned representation of the MMSE equalizer, we obtain

$$
\begin{aligned}
\mathbf{y}[k]= & \frac{1}{\sqrt{M}} \sum_{\mu=0}^{M-1}\left(\mathbf{H}^{H}[\mu] \mathbf{H}[\mu]+\zeta \mathbf{I}_{2}\right)^{-1} \mathbf{H}^{H}[\mu] \\
& \times(\mathbf{H}[\mu] \mathbf{A}[\mu]+\mathbf{N}[\mu]) \mathrm{e}^{j(2 \pi / M) k \mu},
\end{aligned}
$$

with $\mathbf{A}[\mu] \triangleq\left[A_{1}[\mu] A_{2}[\mu]\right]^{T}$ and an i.i.d. frequency domain vector $\mathbf{N}[\mu]$ with independent components of variance $\sigma_{n}^{2}$. Equivalently, $\mathbf{y}[k]$ can be written as

$$
\begin{aligned}
\mathbf{y}[k]= & \frac{1}{\sqrt{M}} \sum_{\mu=0}^{M-1}\left(\mathbf{H}^{H}[\mu] \mathbf{H}[\mu]+\zeta \mathbf{I}_{2}\right)^{-1} \\
& \times\left(\mathbf{H}^{H}[\mu] \mathbf{H}[\mu]+\zeta \mathbf{I}_{2}\right) \mathbf{A}[\mu] \mathrm{e}^{j(2 \pi / M) k \mu} \\
& +\frac{1}{\sqrt{M}} \sum_{\mu=0}^{M-1}\left(\mathbf{H}^{H}[\mu] \mathbf{H}[\mu]+\zeta \mathbf{I}_{2}\right)^{-1} \\
& \times\left(\mathbf{H}^{H}[\mu] \mathbf{N}[\mu]-\zeta \mathbf{I}_{2} \mathbf{A}[\mu]\right) \mathrm{e}^{j(2 \pi / M) k \mu} .
\end{aligned}
$$

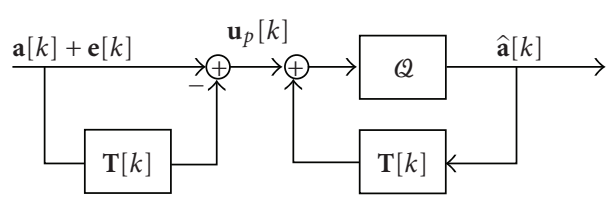

Figure 2: Structure of MIMO DFE receiver.

Thus, the error vector of MMSE equalization is given by

$$
\begin{aligned}
\mathbf{e}[k]= & \frac{1}{\sqrt{M}} \sum_{\mu=0}^{M-1}\left(\mathbf{H}^{H}[\mu] \mathbf{H}[\mu]+\zeta \mathbf{I}_{2}\right)^{-1} \\
& \times\left(\mathbf{H}^{H}[\mu] \mathbf{N}[\mu]-\zeta \mathbf{I}_{2} \mathbf{A}[\mu]\right) \mathrm{e}^{j(2 \pi / M) k \mu}
\end{aligned}
$$

Taking into account the statistical independence of terms for different discrete frequencies $\mu$ in the sum of the right hand side of (9) and the mutual independence of $\mathbf{A}[\mu]$ and $\mathbf{N}[\mu]$, the error correlation matrix can be expressed as

$$
\begin{aligned}
\boldsymbol{\Phi}_{e e}= & \&\left\{\mathbf{e}[k] \mathbf{e}^{H}[k]\right\} \\
= & \frac{1}{M} \sum_{\mu=0}^{M-1}\left(\mathbf{H}^{H}[\mu] \mathbf{H}[\mu]+\zeta \mathbf{I}_{2}\right)^{-1} \\
& \times\left(\sigma_{n}^{2} \mathbf{H}^{H}[\mu] \mathbf{H}[\mu]+\zeta^{2} \sigma_{a}^{2} \mathbf{I}_{2}\right)\left(\mathbf{H}^{H}[\mu] \mathbf{H}[\mu]+\zeta \mathbf{I}_{2}\right)^{-1} \\
= & \frac{\sigma_{n}^{2}}{M} \sum_{\mu=0}^{M-1}\left(\mathbf{H}^{H}[\mu] \mathbf{H}[\mu]+\zeta \mathbf{I}_{2}\right)^{-1} .
\end{aligned}
$$

After MMSE-LE, a bias which is characteristical for MMSE filtering (This bias arises because the error signal $e_{i}[k]$ contains a part depending on $a_{i}[k]$.) is removed and soft output for subsequent channel decoding is calculated from the equalized symbols $y_{i}[k][4]$.

In case of additional beamforming, $\mathbf{H}[\mu]$ has to be replaced by the overall transfer matrix $\mathbf{H}[\mu] \mathbf{P}[\mu]$ in all expressions for MMSE filter and error covariance matrix calculation.

\section{MMSE-DFE for SC-FDMA}

To enhance the performance of MMSE-LE, a MIMO noise (error) prediction-error filter may be inserted after the MMSE linear equalizer as shown in Figure 2 and applied to $\mathbf{y}[k] \triangleq\left[y_{1}[k] y_{2}[k]\right]^{T}, \mathbf{y}[k]=\mathbf{a}[k]+\mathbf{e}[k], \mathbf{a}[k] \triangleq$ $\left[a_{1}[k] a_{2}[k]\right]^{T}$. The introduced postcursor intersymbol interference is removed by decision feedback after the quantizer $\mathcal{Q}$ producing decisions $\hat{\mathbf{a}}[k]$ for $\mathbf{a}[k]$, resulting in an MMSE-DFE structure, where the feedback filter coefficient matrices are identical to those of the prediction filter $\mathbf{T}[k]$, compare with, for example, [5].

The signal after prediction-error filtering is described by

$$
\mathbf{u}_{p}[k]=\mathbf{T}_{e}[k] \otimes \mathbf{a}[k]+\mathbf{w}_{p}[k],
$$


where $\mathbf{T}_{e}[k]$ are the coefficients of the prediction-error filter, $\mathbf{T}_{e}[0]=\mathbf{I}_{2}, \mathbf{T}_{e}[k]=-\mathbf{T}[k], k \in\left\{1,2, \ldots, q_{p}\right\}(\mathbf{T}[k]:$ predictor coefficient matrices, $q_{p}$ : predictor order), $\mathbf{T}_{e}[k]=$ $\mathbf{0}_{2 \times 2}, k \in\left\{q_{p}+1, \ldots, M-1\right\}$, and $\mathbf{w}_{p}[k]$ is the error signal of the MMSE-LE output filtered with the prediction-error filter.

The optimum predictor coefficients are obtained from the multichannel Yule Walker equations $[4,5]$

$$
\begin{aligned}
& {\left[\begin{array}{cccc}
\mathbf{A}[0] & \mathbf{A}[1] & \cdots & \mathbf{A}\left[q_{p}-1\right] \\
\mathbf{A}[-1] & \mathbf{A}[0] & \cdots & \mathbf{A}\left[q_{p}-2\right] \\
\vdots & \vdots & \ddots & \mathbf{A}[1] \\
\mathbf{A}\left[-q_{p}+1\right] & \mathbf{A}\left[-q_{p}+2\right] & \cdots & \mathbf{A}[0]
\end{array}\right]\left[\begin{array}{c}
\mathbf{T}^{H}[1] \\
\mathbf{T}^{H}[2] \\
\vdots \\
\mathbf{T}^{H}\left[q_{p}\right]
\end{array}\right]}
\end{aligned}
$$

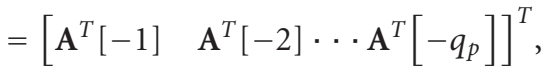

with the cyclic autocorrelation matrix sequence of the error signal of MMSE-LE (with corresponding periodical extension)

$$
\mathbf{A}[k]=\frac{\sigma_{n}^{2}}{M} \sum_{\mu=0}^{M-1}\left(\mathbf{H}^{H}[\mu] \mathbf{H}[\mu]+\zeta \mathbf{I}_{2}\right)^{-1} \mathrm{e}^{j(2 \pi / M) k \mu} .
$$

4.1. Case $\left(q_{p}=M-1\right)$. We now consider the limit case of the maximum possible prediction order, $q_{p}=M-1$. Here, from a closer inspection of (12),

$$
\mathbf{A}[k] \otimes \mathbf{T}_{e}^{H}[-k]=\mathbf{0}, \quad k \in\{1,2, \ldots, M-1\}
$$

can be deduced for the optimum prediction-error filter. (For evaluation of the cyclic convolution arising in (14), the matrix sequences are periodically extended beyond the set $k \in\{0,1, \ldots, M-1\}$.) Solving (14) in the frequency domain and taking into account the constraint $\mathbf{T}_{e}[0]=\mathbf{I}_{2}$, the frequency response $\boldsymbol{S}[\mu]$ of the optimum prediction-error filter can be expressed as

$$
\begin{aligned}
\mathbf{S}[\mu]= & \left(\frac{1}{M} \sum_{\lambda=0}^{M-1}\left(\mathbf{H}^{H}[\lambda] \mathbf{H}[\lambda]+\zeta \mathbf{I}_{2}\right)\right)^{-1} \\
& \cdot\left(\mathbf{H}^{H}[\mu] \mathbf{H}[\mu]+\zeta \mathbf{I}_{2}\right) .
\end{aligned}
$$

After some further straightforward calculations, the covariance matrix of the prediction error $\mathbf{w}_{p}[k], \boldsymbol{\Phi}_{w_{p} w_{p}} \triangleq$ $\mathcal{E}\left\{\mathbf{w}_{p}[k] \mathbf{w}_{p}^{H}[k]\right\}$, is obtained as

$$
\boldsymbol{\Phi}_{w_{p} w_{p}}=\sigma_{n}^{2}\left(\frac{1}{M} \sum_{\lambda=0}^{M-1}\left(\mathbf{H}^{H}[\lambda] \mathbf{H}[\lambda]+\zeta \mathbf{I}_{2}\right)\right)^{-1}
$$

and its power density spectrum as

$$
\begin{aligned}
\sigma_{n}^{2} & \left(\frac{1}{M} \sum_{\lambda=0}^{M-1}\left(\mathbf{H}^{H}[\lambda] \mathbf{H}[\lambda]+\zeta \mathbf{I}_{2}\right)\right)^{-1} \\
\times & \left(\mathbf{H}^{H}[\mu] \mathbf{H}[\mu]+\zeta \mathbf{I}_{2}\right) \\
& \cdot\left(\frac{1}{M} \sum_{\lambda=0}^{M-1}\left(\mathbf{H}^{H}[\lambda] \mathbf{H}[\lambda]+\zeta \mathbf{I}_{2}\right)\right)^{-1} .
\end{aligned}
$$

The frequency response in (15) may be viewed as that of a multichannel extension of an interpolation-error filter, compare with [12]. This is because for $q_{p}=M-1$ all other available error vectors, that is, future and past vectors, are contributing to the estimation of the current error vector, and the filter no longer acts as a predictor but as an interpolator. Also (16) and (17) may be interpreted as multichannel cyclic generalizations of corresponding results in [12]. It is important to note that an interpolation error is not white, in contrast to the prediction error produced by an optimum causal prediction filter, compare with also [13]. In fact, it can be shown that the cascade of MMSELE and an interpolation-error filter has a frequency response proportional to $\mathbf{H}^{H}[\mu]$, that is, a matched filter results requiring a DFE feedback filter with equally strong causal and noncausal coefficients.

4.2. Case $\left(q_{p}=(M-1) / 2\right)$. In a system with cyclic convolution, a predictor with $q_{p}=(M-1) / 2(M$ odd $)$ may be viewed as the counterpart of a classical, causal prediction filter of infinite order with linear convolution. Therefore, it can be expected that for sufficiently large $M$, results for infinite prediction order and linear convolution hold well for the considered case. In [9], it has been shown that for a multichannel MMSE-DFE, the optimum filters minimizing $\operatorname{tr}\left(\boldsymbol{\Phi}_{w_{p} w_{p}}\right)$ (arithmetic MSE) minimize also $\operatorname{det}\left(\boldsymbol{\Phi}_{w_{p} w_{p}}\right)$ (geometric MSE), that is, both criteria are equivalent, and an expression for the minimum determinant has been given $[9,(37)]$. Adapting this expression to our notation and discretizing the integral,

$$
\begin{aligned}
& \operatorname{det}\left(\boldsymbol{\Phi}_{w_{p} w_{p}}\right) \\
& =\exp \left(\frac{1}{M} \sum_{\mu=0}^{M-1} \ln \left(\operatorname{det}\left(\sigma_{n}^{2}\left(\mathbf{H}^{H}[\mu] \cdot \mathbf{H}[\mu]+\zeta \mathbf{I}_{2}\right)^{-1}\right)\right)\right)
\end{aligned}
$$

is obtained. Elaborating further on (18) yields

$$
\operatorname{det}\left(\boldsymbol{\Phi}_{w_{p} w_{p}}\right)=\left(\sigma_{n}^{2}\right)^{2} \sqrt[M]{\prod_{\mu=0}^{M-1} \operatorname{det}\left(\left(\mathbf{H}^{H}[\mu] \mathbf{H}[\mu]+\zeta \mathbf{I}_{2}\right)^{-1}\right)} .
$$

Again, for the case of additional beamforming $\mathbf{H}[\mu]$ has to be replaced by $\mathbf{H}[\mu] \mathbf{P}[\mu]$ in all expressions. 


\section{Optimum Beamforming and Power Allocation for MMSE-LE}

If knowledge of the MIMO transmission channel is available at the transmitter, this can be exploited to make the transmit signal more robust to distortions during transmission. Therefore, in this section, a beamformer is presented which is optimal in the MMSE sense when MMSE linear equalization is applied at the receiver side.

5.1. Design of MMSE Beamforming Filter. For the design of the beamforming matrices $\mathbf{P}[\mu], \mu \in\{0,1, \ldots, M-1\}$, the error variances $\sigma_{e, i}^{2}, i \in\{1,2\}$, after MMSE-LE are considered. Here, the optimum beamformer is defined as the beamformer minimizing $\sigma_{e, 1}^{2}+\sigma_{e, 2}^{2}$, that is, $\operatorname{tr}\left(\boldsymbol{\Phi}_{e e}\right)$ for a given transmit power. ( An alternative optimization criterion would be the average bit error rate (BER) after MMSE-LE of both transmit streams instead of the sum of MSEs. However, there seems to be no closed-form solution for minimum BER beamforming.) Thus, considering (10) and replacing $\mathbf{H}[\mu]$ by $\mathbf{H}[\mu] \mathbf{P}[\mu]$, the cost function to be minimized can be expressed as

$$
J=\operatorname{tr}\left(\frac{\sigma_{n}^{2}}{M} \sum_{\mu=0}^{M-1}\left(\mathbf{P}^{H}[\mu] \mathbf{H}^{H}[\mu] \mathbf{H}[\mu] \mathbf{P}[\mu]+\zeta \mathbf{I}_{2}\right)^{-1}\right) .
$$

Hence,the optimum beamformer is given by the solution of the optimization problem

$$
\begin{aligned}
\underset{\mathbf{P}[0], \mathbf{P}[1], \ldots, \mathbf{P}[M-1]}{\min } & J \\
\text { s.t. } & \operatorname{tr}\left(\sum_{\mu=0}^{M-1} \mathbf{P}[\mu] \mathbf{P}^{H}[\mu]\right) \leq 2 M \frac{P}{\sigma_{a}^{2}},
\end{aligned}
$$

where $P$ denotes the prescribed average transmit power per subcarrier and i.i.d. data sequences have been assumed for the power constraint. Using the eigenvalue decomposition

$$
\mathbf{H}^{H}[\mu] \mathbf{H}[\mu]=\mathbf{V}[\mu] \boldsymbol{\Lambda}_{H}[\mu] \mathbf{V}^{H}[\mu]
$$

with a $2 \times 2$ unitary matrix $\mathbf{V}[\mu]$ and a diagonal matrix $\boldsymbol{\Lambda}_{H}[\mu]$ with entries $d_{1}^{2}[\mu], d_{2}^{2}[\mu]$ on its main diagonal, where $d_{1}[\mu]$, $d_{2}[\mu]$ are nonnegative, and the property

$$
\operatorname{tr}\left(\left(\mathbf{I}_{X}+\mathbf{A B}\right)^{-1}\right)=\operatorname{tr}\left(\left(\mathbf{I}_{X}+\mathbf{B A}\right)^{-1}\right)
$$

for square matrices $\mathbf{A}$ and $\mathbf{B}$, we obtain

$$
\begin{array}{r}
J=\frac{\sigma_{n}^{2}}{M \zeta} \sum_{\mu=0}^{M-1} \operatorname{tr}\left(\left(\frac{1}{\zeta} \boldsymbol{\Lambda}_{H}^{1 / 2}[\mu] \mathbf{V}^{H}[\mu] \mathbf{P}[\mu] \mathbf{P}^{H}[\mu]\right.\right. \\
\left.\left.\times \mathbf{V}[\mu] \boldsymbol{\Lambda}_{H}^{1 / 2}[\mu]+\mathbf{I}_{2}\right)^{-1}\right),
\end{array}
$$

compare with also [14], where an OFDM MMSE beamforming problem has been considered. Inserting the singular value decomposition (SVD) of $\mathbf{P}[\mu]$,

$$
\mathbf{P}[\mu]=\mathbf{L}[\mu] \boldsymbol{\Lambda}_{P}[\mu] \mathbf{K}^{H}[\mu],
$$

with $2 \times 2$ unitary matrices $\mathbf{L}[\mu], \mathbf{K}[\mu]$ and diagonal matrix $\Lambda_{P}[\mu]$ with nonnegative entries $c_{1}[\mu], c_{2}[\mu]$ on its main diagonal, into (24) yields

$$
\begin{aligned}
J= & \frac{\sigma_{n}^{2}}{M \zeta} \\
& \times \sum_{\mu=0}^{M-1} \operatorname{tr}\left(\left(\frac{1}{\zeta} \Lambda_{H}^{1 / 2}[\mu] \mathbf{C}[\mu] \Lambda_{P}^{2}[\mu] \mathbf{C}^{H}[\mu] \Lambda_{H}^{1 / 2}[\mu]+\mathbf{I}_{2}\right)^{-1}\right)
\end{aligned}
$$

with $\mathbf{C}[\mu] \triangleq \mathbf{V}^{H}[\mu] \mathbf{L}[\mu] . \mathbf{K}[\mu]$ has an influence neither on the cost function nor on the power constraint in (21). Therefore, $\mathbf{K}[\mu]=\mathbf{I}_{2}$ can be chosen without any loss of generality. However, $\mathbf{K}[\mu]$ has an influence on the peak power and can be used to reduce the PAPR of the transmit signal, compare with Section 7 . The matrix $\mathbf{U}[\mu] \triangleq$ $\Lambda_{H}^{1 / 2}[\mu] \mathbf{C}[\mu] \Lambda_{P}^{2}[\mu] \mathbf{C}^{H}[\mu] \Lambda_{H}^{1 / 2}[\mu]$ in (26) is Hermitian and an eigenvalue decomposition

$$
\mathbf{U}[\mu]=\mathbf{Q}[\mu] \Lambda_{U}[\mu] \mathbf{Q}^{H}[\mu]
$$

exists with a $2 \times 2$ unitary matrix $\mathbf{Q}[\mu]$. Then, the cost function can be written as

$$
J=\frac{\sigma_{n}^{2}}{M \zeta} \sum_{\mu=0}^{M-1} \operatorname{tr}\left(\left(\frac{1}{\zeta} \mathbf{Q}[\mu] \boldsymbol{\Lambda}_{U}[\mu] \mathbf{Q}^{H}[\mu]+\mathbf{I}_{2}\right)^{-1}\right) .
$$

In (28), $J$ is influenced only by the matrix of eigenvalues $\Lambda_{U}[\mu]$ but not by the modal matrix $\mathbf{Q}[\mu]$. Therefore, we restrict ourselves to beamformers with $\mathbf{Q}[\mu]=\mathbf{I}_{2}$ which implies that $\mathbf{U}[\mu]$ is diagonal and $\mathbf{C}[\mu]=\mathbf{I}_{2}$, that is, $\mathbf{L}[\mu]=$ $\mathbf{V}[\mu]$, corresponding to an eigenbeamforming solution. In fact, for any beamforming filter resulting in matrices $\mathbf{U}[\mu]$ according to (27) an equivalent eigenbeamforming filter $\mathbf{P}_{\text {eig }}[\mu]$ with SVD matrices $\mathbf{L}_{\text {eig }}[\mu]=\mathbf{V}[\mu]$ and $\boldsymbol{\Lambda}_{P, \text { eig }}[\mu]=$ $\boldsymbol{\Lambda}_{H}^{-1 / 2}[\mu] \boldsymbol{\Lambda}_{U}^{1 / 2}[\mu]$ exists resulting in the same cost function. Now it remains to be shown that the eigenbeamforming solution does not affect the power constraint. For this, we consider $\mathbf{P}[\mu] \mathbf{P}^{H}[\mu]$ in $(21)$,

$$
\begin{aligned}
& \operatorname{tr}\left(\mathbf{P}[\mu] \mathbf{P}^{H}[\mu]\right) \\
& =\operatorname{tr}\left(\mathbf{V}[\mu] \mathbf{V}^{H}[\mu] \mathbf{P}[\mu] \mathbf{P}^{H}[\mu]\right) \\
& =\operatorname{tr}\left(\mathbf{V}^{H}[\mu] \mathbf{P}[\mu] \mathbf{P}^{H}[\mu] \mathbf{V}[\mu]\right) \\
& =\operatorname{tr}\left(\boldsymbol{\Lambda}_{H}^{-1 / 2}[\mu] \boldsymbol{\Lambda}_{H}^{1 / 2}[\mu] \mathbf{V}^{H}[\mu] \mathbf{P}[\mu] \mathbf{P}^{H}[\mu]\right. \\
& \left.\quad \times \mathbf{V}[\mu] \boldsymbol{\Lambda}_{H}^{1 / 2}[\mu] \boldsymbol{\Lambda}_{H}^{-1 / 2}[\mu]\right) \\
& =\operatorname{tr}\left(\boldsymbol{\Lambda}_{H}^{-1}[\mu] \mathbf{Q}[\mu] \boldsymbol{\Lambda}_{U}[\mu] \mathbf{Q}^{H}[\mu]\right) \\
& \geq \operatorname{tr}\left(\boldsymbol{\Lambda}_{H}^{-1}[\mu] \boldsymbol{\Lambda}_{U}[\mu]\right)=\operatorname{tr}\left(\boldsymbol{\Lambda}_{P, \mathrm{eig}}^{2}[\mu]\right) \\
& =\operatorname{tr}\left(\mathbf{P}_{\mathrm{eig}}[\mu] \mathbf{P}_{\mathrm{eig}}^{H}[\mu]\right),
\end{aligned}
$$

where $\operatorname{tr}\left(\begin{array}{ll}\mathbf{A} & \mathbf{B}\end{array}\right)=\operatorname{tr}\left(\begin{array}{ll}\mathbf{B} & \mathbf{A}\end{array}\right)$ has been used and the step from (29) to (30) follows from majorization theory, compare with 
$[14,15]$. Hence, we have proved that there is always an eigenbeamformer which exhibits the same cost function as a given arbitrary beamformer at equal or even lower transmit power. Therefore, eigenbeamforming is optimum and considered further in the following.

5.2. MSE Minimizing Power Allocation for Eigenbeamforming. For eigenbeamforming, it is straightforward to show that the error correlation matrix in (10) is given by

$$
\boldsymbol{\Phi}_{e e}=\frac{\sigma_{n}^{2}}{M} \sum_{\mu=0}^{M-1} \operatorname{diag}\left\{\frac{1}{p_{1}[\mu] d_{1}^{2}[\mu]+\zeta}, \frac{1}{p_{2}[\mu] d_{2}^{2}[\mu]+\zeta}\right\},
$$

where $p_{i}[\mu] \triangleq c_{i}^{2}[\mu]$ is the power allocation coefficient for transmit antenna $i$ and subcarrier $\mu$. Optimization problem (21) simplifies for eigenbeamforming to

$$
\begin{aligned}
\min _{p_{1}[\cdot], p_{2}[\cdot]} & \frac{\sigma_{n}^{2}}{M} \sum_{\mu=0}^{M-1} \sum_{i=1}^{2} \frac{1}{p_{i}[\mu] d_{i}^{2}[\mu]+\zeta} \\
\text { s.t. } & p_{i}[\mu] \geq 0 \quad \forall \mu, i \in\{1,2\}, \\
& \sum_{\mu=0}^{M-1} \sum_{i=1}^{2} p_{i}[\mu]=2 M \frac{P}{\sigma_{a}^{2}} .
\end{aligned}
$$

Convex optimization problems of the form (32) have been considered for example, in $[16,17]$. Via the Karush-KuhnTucker (KKT) optimality conditions [16], the following solution can be obtained:

$$
\begin{aligned}
p_{i}[\mu]= & \frac{2 M P / \sigma_{a}^{2}+\zeta \sum_{i=1}^{2} \sum_{\lambda \in \delta_{i}} 1 / d_{i}^{2}[\lambda]}{\sum_{i=1}^{2} \sum_{\lambda \in \vartheta_{i}}\left(1 / d_{i}[\lambda]\right)} \frac{1}{d_{i}[\mu]} \\
& -\zeta \frac{1}{d_{i}^{2}[\mu]}, \quad i \in\{1,2\}, \quad \mu \in 8_{i}, \\
p_{i}[\mu]= & 0, \quad i \in\{1,2\}, \quad \mu \notin \wp_{i} .
\end{aligned}
$$

Here, the subsets $\delta_{i} \subseteq\{0,1, \ldots, M-1\}$ are determined as follows. First, for each $i$ the indices $\mu$ with $d_{i}[\mu]=0$ are determined and deleted from $\{0,1, \ldots, M-1\}$ to have initial choices for $\delta_{i}$. Then, if some $p_{i}[\mu]$ according to (33) are negative, the smallest value $d_{i}[\mu], i \in\{1,2\}, \mu \in \delta_{i}$ is determined and the corresponding subcarrier index $\mu$ is deleted from the respective subset $\delta_{i}$. This procedure is repeated until all $p_{i}[\mu]$ according to (33) are nonnegative. The resulting coefficients may be viewed as a modified waterfilling solution for LE, in contrast to the classical capacity-achieving waterfilling solution [17].

5.3. Further Discussion. By setting $\zeta=0$ in all derivations for beamforming for MMSE-LE, corresponding results for zero-forcing (ZF) LE can be obtained as a special case in a straightforward way. The optimum power allocation factors for eigenbeamforming are then given by $p_{i}[\mu]=C / d_{i}[\mu]$ with some constant $C$ and $\delta_{i}=\{0,1, \ldots, M-1\}, i \in\{1,2\}$, compare with (33). Note that $d_{i}[\mu]>0$, for all $i$, for all $\mu$ has to be fulfilled as a condition for the existence of a stable $\mathrm{ZF}$ equalizer. Thus, the frequency response of the beamforming filter is given by

$$
\mathbf{P}[\mu]=\mathbf{V}[\mu] \cdot \operatorname{diag}\left\{\frac{\sqrt{C}}{\sqrt{d_{1}[\mu]}}, \frac{\sqrt{C}}{\sqrt{d_{2}[\mu]}}\right\} .
$$

Using an SVD of $\mathbf{H}[\mu]$, it is straightforward to show that the frequency response of the ZF linear equalizer, $\mathrm{F}_{\mathrm{ZF}-\mathrm{LE}}[\mu]=$ $(\mathbf{H}[\mu] \mathbf{P}[\mu])^{-1}$, can be expressed as

$$
\mathbf{F}_{\mathrm{ZF}-\mathrm{LE}}[\mu]=\operatorname{diag}\left\{\frac{1 / \sqrt{C}}{\sqrt{d_{1}[\mu]}}, \frac{1 / \sqrt{C}}{\sqrt{d_{2}[\mu]}}\right\} \cdot \mathbf{M}^{H}[\mu],
$$

where $\mathbf{M}[\mu]$ is a unitary matrix. It can be observed that factors $1 / \sqrt{d_{i}[\mu]}$ are employed for both beamforming filtering and ZF-LE, that is, beamforming acts as a kind of preequalization and the channel equalizer is split in equal (up to a scaling and unitary matrices) transmitter and receiver parts.

It is interesting to note that our results for beamforming for SC-FDMA with LE are similar in spirit to the classical results of Berger and Tufts [8] and Yang and Roy [18] who developed the optimum continuous-time transmit filters assuming LE at the receiver for transmission with conventional linear modulation over single-input single-output (SISO) and MIMO channels, respectively.

The computational complexity of beamforming filter calculation is governed by the complexity of the eigenvalue decompositions of $M$ matrices $\mathbf{H}^{H}[\mu] \mathbf{H}[\mu]$ of size $2 \times 2$ $(\mathcal{O}(M))$ and by the number of iterations needed to find the optimum coefficients $p_{i}[\mu], i \in\{1,2\}$ according to (33) and (34).

\section{Optimum Beamforming and Power Allocation for MMSE-DFE}

6.1. Case $\left(q_{p}=M-1\right)$. First, we consider the maximum possible prediction order and replace $\mathbf{H}[\mu]$ by $\mathbf{H}[\mu] \mathbf{P}[\mu]$ in (16). Assuming an eigenbeamforming solution, $\mathbf{P}[\mu]=$ $\mathrm{V}[\mu] \Lambda_{P}[\mu]$, with a diagonal matrix $\boldsymbol{\Lambda}_{P}[\mu]$ with nonnegative entries $c_{1}[\mu], c_{2}[\mu]$ on its main diagonal, the sum of signalto-prediction-error ratios of both data streams can be written as

$$
\frac{\sigma_{a}^{2}}{\sigma_{w_{p, 1}}^{2}}+\frac{\sigma_{a}^{2}}{\sigma_{w_{p, 2}}^{2}}=\frac{\sigma_{a}^{2}}{M \sigma_{n}^{2}} \sum_{\mu=0}^{M-1} \sum_{i=1}^{2}\left(p_{i}[\mu] d_{i}^{2}[\mu]+\zeta\right),
$$

where $p_{i}[\mu] \triangleq c_{i}^{2}[\mu]$ is again the power allocation coefficient for transmit antenna $i$ and subcarrier $\mu$. It is easy to see that an optimum power allocation policy puts all available transmit power in that stream $i$ and subcarrier $\mu$ with maximum $d_{i}^{2}[\mu]$. This, however, results in a widely spread impulse response of overall channel and corresponding DFE feedback filter, that is, the MMSE-DFE is likely to be affected by severe error propagation. It should be noted that such a feedback filter fed by hard decisions can be only 
employed in the last iterations of an iterative DFE, when reliable past and future decisions are available [19]. However, beamforming should be adjusted to the situation in the first iteration where a causal feedback filter has to be applied. Because of this and other practical constraints, the scheme with $q_{p}=M-1$ is mainly of theoretical interest and not considered for our numerical results for noniterative DFE schemes (Iterative DFE schemes are beyond the scope of this paper).

6.2. Case $\left(q_{p}=(M-1) / 2\right)$. With a transmit power constraint the optimum beamformer minimizing the geometric MSE (19) of MMSE-DFE is given by the solution of the optimization problem

$$
\begin{aligned}
\min _{\mathbf{P}[0], \mathbf{P}[1], \ldots, \mathbf{P}[M-1]} & J \\
\text { s.t. } & \operatorname{tr}\left(\sum_{\mu=0}^{M-1} \mathbf{P}[\mu] \mathbf{P}^{H}[\mu]\right) \leq 2 M \frac{P}{\sigma_{a}^{2}},
\end{aligned}
$$

where the cost function $J$ is given by

$$
J=\prod_{\mu=0}^{M-1} \operatorname{det}\left(\left(\mathbf{P}^{H}[\mu] \mathbf{H}^{H}[\mu] \mathbf{H}[\mu] \mathbf{P}[\mu]+\zeta \mathbf{I}_{2}\right)^{-1}\right)
$$

and $P$ denotes again the prescribed average transmit power per subcarrier.

In [17, pages 136-137], it has been shown that for problems of the form (38), (39), eigenbeamforming, $\mathbf{P}[\mu]=$ $\mathrm{V}[\mu] \boldsymbol{\Lambda}_{P}[\mu]$, is optimum, resulting in the power allocation task

$$
\begin{aligned}
\min _{p_{1}[\cdot], p_{2}[\cdot]} & \prod_{\mu=0}^{M-1} \prod_{i=1}^{2} \frac{1}{p_{i}[\mu] d_{i}^{2}[\mu]+\zeta} \\
\text { s.t. } & p_{i}[\mu] \geq 0 \quad \forall \mu, i \in\{1,2\}, \\
& \sum_{\mu=0}^{M-1} \sum_{i=1}^{2} p_{i}[\mu]=2 M \frac{P}{\sigma_{a}^{2}} .
\end{aligned}
$$

Via the Karush-Kuhn-Tucker (KKT) optimality conditions [16], the well-known classical waterfilling solution is obtained,

$$
\begin{gathered}
p_{i}[\mu]=\left(\omega-\zeta \frac{1}{d_{i}^{2}[\mu]}\right), \quad i \in\{1,2\}, \mu \in s_{i}, \\
p_{i}[\mu]=0, \quad i \in\{1,2\}, \mu \notin \delta_{i} .
\end{gathered}
$$

The determination of water level $\omega$ and subsets $\delta_{i} \subseteq$ $\{0,1, \ldots, M-1\}$ is well investigated, compare with for example, [17]. It should be noted that the cost function $J$ in (39) characterizes the MMSE-DFE performance exactly only for $M \rightarrow \infty$ and $q_{p}=(M-1) / 2$, however, it is still a very good performance approximation for practically relevant $M$ and $q_{p}$ and therefore suitable for beamformer optimization also in these cases.

Unlike linear equalization, the capacity-achieving waterfilling power allocation solution is obtained for MMSE-DFE, which is in agreement with results for systems with linear convolution, compare with for example, [9]. It should be noted that for linear equalization power has to be allocated mainly to subcarriers where the channel frequency response is weak, whereas for DFE mainly the strong subcarriers are used. Only for $\sigma_{n}^{2} \rightarrow 0$, a flat transmit spectrum results.

Regarding the computational complexity of beamforming filter calculation, similar remarks as for LE hold, compare with last paragraph of Section 5.

\section{PAPR Reduction}

In the previous analysis, we chose for simplicity $\mathbf{K}[\mu]$ in (25) to be the identity matrix. As a unitary $\mathbf{K}[\mu]$ has no influence on the cost functions (20) and (39) and the power constraint in (38), in this section $\mathbf{K}[\mu]$ is exploited for PAPR reduction. For this purpose the SLM method, proposed in [10] and extended for MIMO systems in [11], is invoked and adjusted to our problem. First, we define $N_{\text {set }}$ subsets of subcarriers $U_{\iota}, \iota=1, \ldots, N_{\text {set }}$, where $U_{\iota}$ contains a specified subcarrier arrangement for both transmit antennas. Subsequently, a phase rotation $\theta_{l} \in \Theta$, where $\Theta$ contains $N_{\theta}$ allowed rotation angles, is included into the beamforming filter for each subset $U_{l}$, exploiting unused degrees of freedom in filter design, compare with Section 5.1. Thus, the modified MMSE optimum eigenbeamformer for $\mu \in U_{\iota}$ is given by

$$
\begin{gathered}
\mathbf{P}[\mu]=\mathbf{V}[\mu] \operatorname{diag}\left\{\sqrt{p_{1}[\mu]}, \sqrt{p_{2}[\mu]}\right\} \mathbf{K}_{\mathrm{rot}}^{H}[\mu], \\
\text { with } \quad \mathbf{K}_{\mathrm{rot}}[\mu]=\left[\begin{array}{cc}
\cos \left(\theta_{l}\right) & -\sin \left(\theta_{l}\right) \\
\sin \left(\theta_{\iota}\right) & \cos \left(\theta_{l}\right)
\end{array}\right],
\end{gathered}
$$

where $\mathbf{K}_{\mathrm{rot}}[\mu]$ is the adopted unitary rotation matrix, $p_{1}[\mu]$ and $p_{2}[\mu]$ are the MMSE power allocation coefficients according to (33) and (34) or (41), depending on whether MMSE-LE or MMSE-DFE is used, and $\mathrm{V}[\mu]$ is the unitary matrix obtained by the eigenvalue decomposition (22). We choose that combination of $\theta_{l}$ s that minimizes the PAPR defined as

$$
\operatorname{PAPR}=\frac{\max _{i, \kappa}\left\{\left|b_{i}[\kappa]\right|^{2}\right\}}{(1 / 2 N) \sum_{i=1}^{2} \sum_{\kappa=0}^{N-1}\left|b_{i}[\kappa]\right|^{2}},
$$

which is calculated for every combination of rotation angles $\theta_{\iota}$ in time domain. This procedure is repeated for each SC-FDMA symbol. Note that with increasing number of angles in $\Theta$ and increasing number of subsets, the number of possible combinations increases according to $N_{\theta}^{N_{\text {set }}}$ and, hence, the computational complexity to find the best $\theta_{l}$ s increases. In order to take into account the rotation operation in equalizer design at the receiver side appropriately, the $N_{\text {set }}$ chosen $\theta_{l}$ s have to be transmitted to the receiver as side information, as is typically done in SLM type of PAPR reduction schemes, compare with $[10,11]$. 


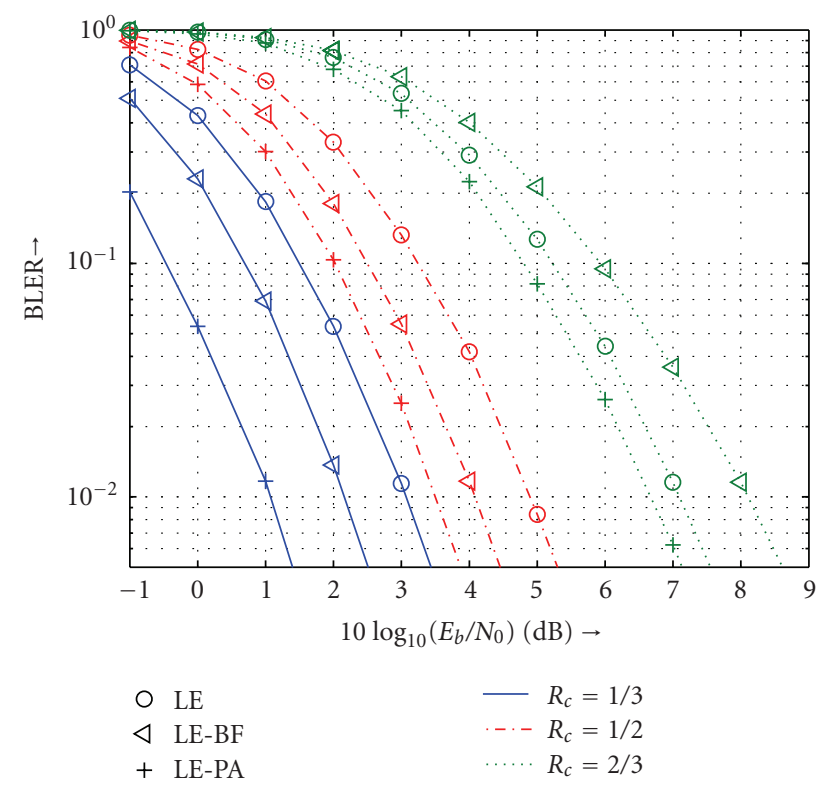

FIgUre 3: BLER for linear MMSE equalization (LE), LE with beamforming (LE-BF), and LE with beamforming and power allocation (LE-PA) for Pedestrian B channel.

\section{Numerical Results}

8.1. Assumptions for Simulations. For the presented simulation results, parameters based on the LTE FDD standard [1] are adopted. Here, Turbo coding with code rate $R_{c}$ and following channel interleaving is applied over a block of two slots, each containing 7 SC-FDMA symbols. For parallel transmission each of the two slots is assigned to one antenna. The DFT sizes are chosen to $M=300$ and $N=512$, where $v_{0}=60$. The MIMO subchannels are assumed to be mutually independent, and the receivers and transmitters with beamforming have ideal channel knowledge.

8.2. Results for MMSE-LE. Figures 3 and 4 show the block error rate (BLER) after channel decoding versus $E_{b} / N_{0}$ ( $E_{b}$ : average received bit energy, $N_{0}$ : single-sided power spectral density of the continuous-time noise) for code rates $R_{c} \in\{1 / 3,1 / 2,2 / 3\}$ and for transmission over a MIMO channel with ITU Pedestrian B and A subchannels [1], respectively. For each channel type, the performance of conventional linear equalization without beamforming (LE), linear equalization with eigenbeamforming and uniform power allocation (LE-BF), and linear equalization with eigenbeamforming and optimal power allocation (LE-PA) is shown. For $R_{c}=1 / 3$ and $R_{c}=1 / 2$ a significant gain can be achieved by applying LE-BF only, but using additionally the proposed power allocation yields a further performance improvement for both channels. However, for $R_{c}=2 / 3$ we observe a degradation of LE-BF relative to the BLER of LE for both channel profiles. By applying the optimal power allocation, the loss introduced by eigenbeamforming can be compensated, showing better results than LE. To investigate this behaviour more in detail the MSEs of the substreams

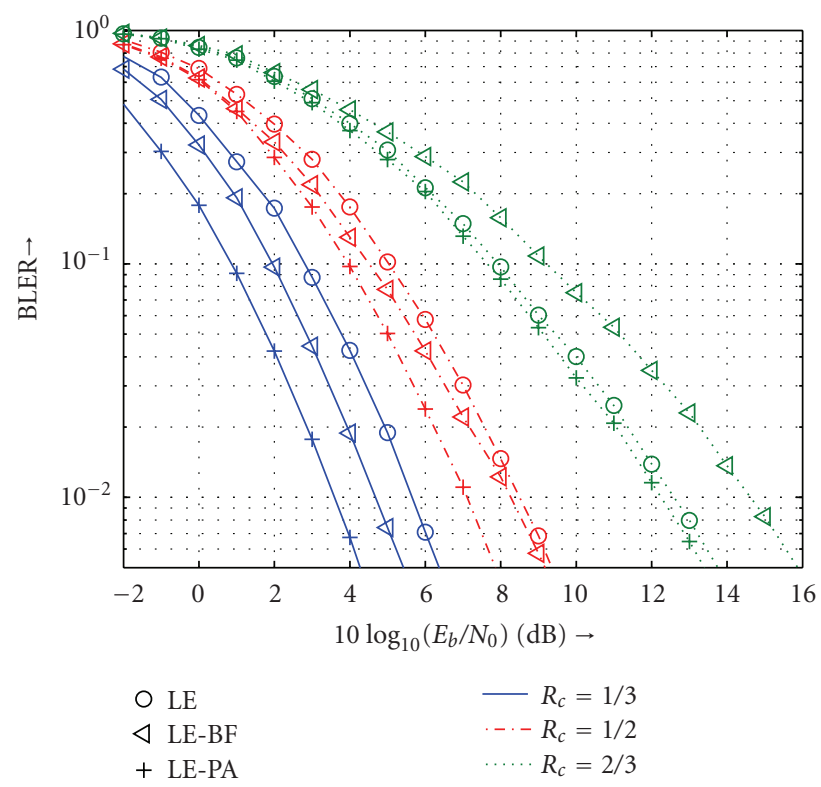

FIGURE 4: BLER for linear MMSE equalization (LE), LE with beamforming (LE-BF), and LE with beamforming and power allocation (LE-PA) for Pedestrian A channel.

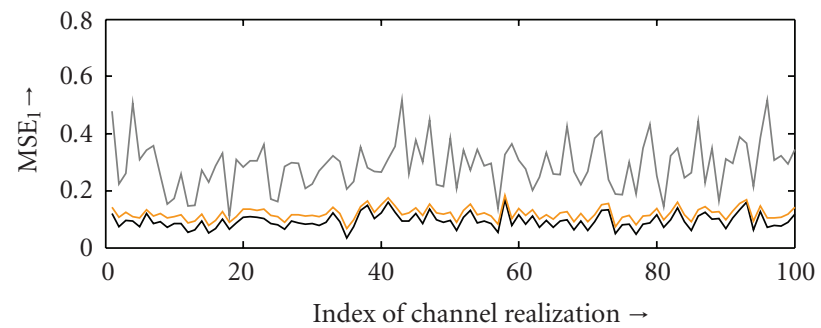

(a)

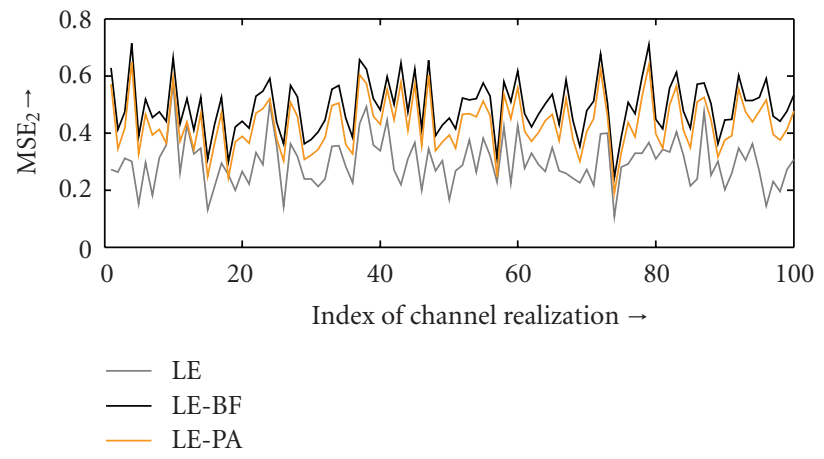

(b)

Figure 5: MSE at $E_{b} / N_{0}=7 \mathrm{~dB}$ for LE, LE-PA, and LE-BF for transmit antenna 1 (a) and transmit antenna 2 (b), respectively, for different realizations of Pedestrian $\mathrm{B}$ channel impulse responses.

are analyzed. Figure 5 shows the MSE of LE, LE-PA, and LE-BF for transmit antenna 1 (Figure 5(a)) and transmit antenna 2 (Figure 5(b)), respectively. The MSE is captured over a set of realizations of the Pedestrian $B$ channel at $E_{b} / N_{0}=7 \mathrm{~dB}$, and depicted versus the index of the channel realization. For calculation of the MSE of a given channel 


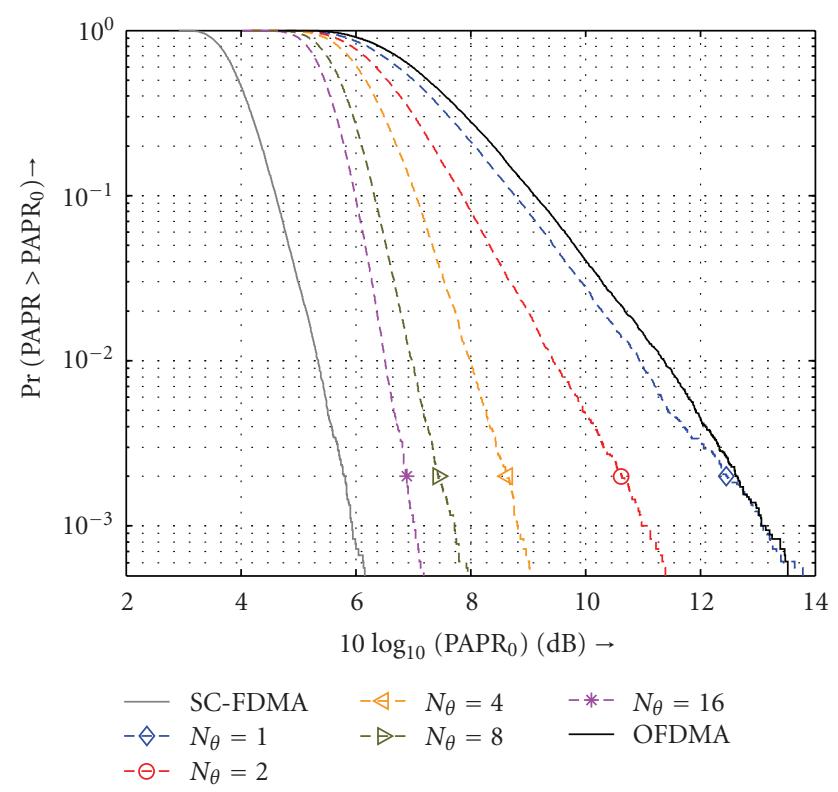

FIgURE 6: PAPR for SC-FDMA transmission without power allocation (solid gray), SC-FDMA transmission with beamforming and power allocation for $\operatorname{LE}\left(E_{b} / N_{0}=7 \mathrm{~dB}\right)$ (dash-dotted), and OFDMA transmission (solid black) for a Pedestrian B channel profile.

realization, (10) has been used, where $\mathbf{H}[\mu]$ has been replaced by $\mathbf{H}[\mu] \mathbf{P}[\mu]$ in case of beamforming. Compared to the MSE of LE, eigenbeamforming reduces the MSE for transmit antenna 1 significantly while for transmit antenna 2 the MSE is boosted, that is, there is always one strong subchannel with reliable transmission and one weak subchannel delivering unreliable symbols. If Turbo coding with low code rates is employed, this improves the BLER as for strong encoding there are sufficiently many reliable symbols to perform error correction. However, for $R_{c}=2 / 3$, with the same amount of reliable information the decoder is not able to recover all symbols well. If MSE optimal power allocation is applied, the gap between the two subchannels is reduced, allowing a higher MSE at transmit antenna 1 and reducing the MSE at transmit antenna 2. Hence, the degradation introduced by eigenbeamforming is compensated so that LE-PA gives the best results for the considered code rates.

Finally, the PAPR of the proposed beamforming scheme is investigated. For simulations, the $N_{\text {set }}=2$ subsets are defined by $U_{1}=\{\mu=0,1, \ldots, M / 2-1\}$ and $U_{2}=$ $\{\mu=M / 2, M / 2+1, \ldots, M-1\}$ and the considered angles of rotation are given by $\Theta=\left\{2 k \pi / N_{\theta} \mid k=0,1, \ldots, N_{\theta}-1\right\}$. Note, that $N_{\theta}=1$ corresponds to MMSE beamforming without any rotation operation. Figure 6 shows the complementary cumulative density function of the PAPR for SC-FDMA transmission without beamforming, with MMSE beamforming for $\operatorname{LE}\left(E_{b} / N_{0}=7 \mathrm{~dB}\right)$ and different $N_{\theta}$, and OFDMA transmission. As is well known, pure SC-FDMA transmission has a lower PAPR than OFDMA transmission which can also be observed here. But applying MMSE

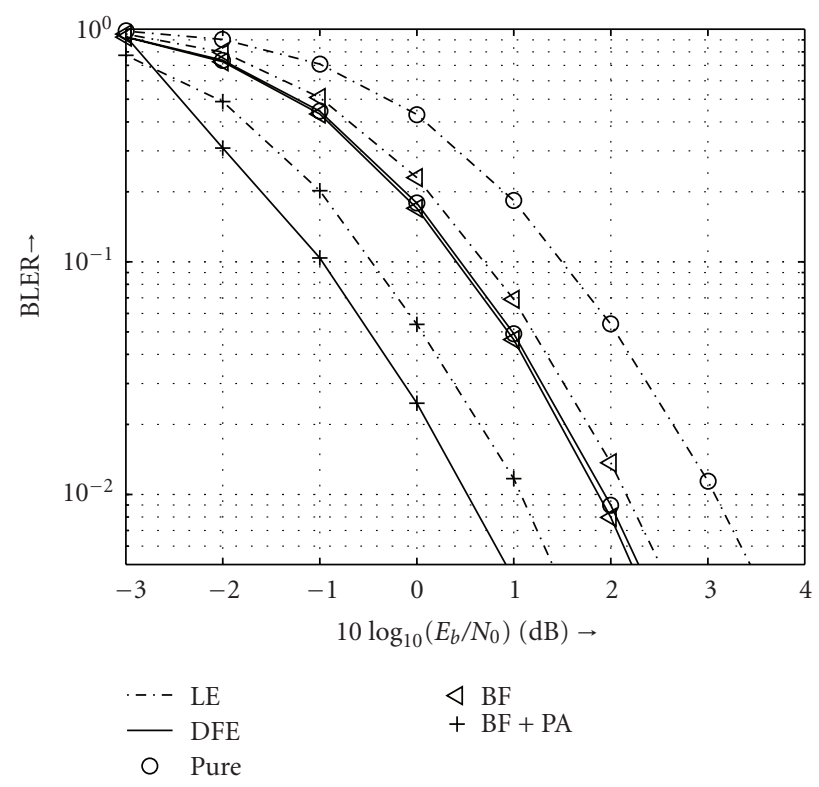

FIGURE 7: BLER for conventional MMSE-LE (dash-dotted line, "o"), MMSE-LE with eigenbeamforming (dash-dotted line, “ $\triangleleft$ ”), MMSE-LE with eigenbeamforming and MMSE power allocation (dash-dotted line, “+”), conventional DFE (solid line, “o"), DFE with eigenbeamforming (solid line, “ $\triangleleft$ ”), and DFE with eigenbeamforming and MMSE power allocation (solid line, “+”). Pedestrian B channel profile.

beamforming with $N_{\theta}=1$ increases the PAPR, nearly bridging the gap between OFDMA and SC-FDMA. With increasing $N_{\theta}$ the PAPR for MMSE beamforming can be decreased, where we note that additional PAPR reduction is diminishing for $N_{\theta}>8$. From Figure 6 we can see that already $N_{\theta}=4$ is sufficient to reduce the PAPR significantly, also meaning that the increase in computational complexity due to the proposed PAPR reduction scheme can be kept low. Recall, that in contrast to symbol amplitude clipping as considered in [7], the proposed PAPR reduction technique does not have any effects on the BLER performance.

8.3. Results for MMSE-DFE. For DFE a code rate of $R_{c}=1 / 3$ is used, and $q_{b}=60$ symbols are fed back, where we assume ideal feedback. Note that the performance of DFE with ideal feedback can be achieved with Tomlinson-Harashima precoding (up to a small transmit power increase) [20] or alternatively by an interleaving scheme that allows the use of decoded bits to generate the feedback symbols $[5,21]$.

Figures 7 and 8 show the BLER after channel decoding versus $E_{b} / N_{0}$ for different channel profiles. Hereby, the performance of DFE without beamforming, DFE with eigenbeamforming, and DFE with eigenbeamforming and MMSE power allocation is compared to that of the conventional LE, LE with eigenbeamforming, and LE with MMSE beamforming, respectively.

For the simulation results shown in Figure 7, the Pedestrian B channel profile has been used for the MIMO subchannels. It can be seen clearly, that each of the DFE schemes exhibits a lower BLER than the corresponding LE scheme and 


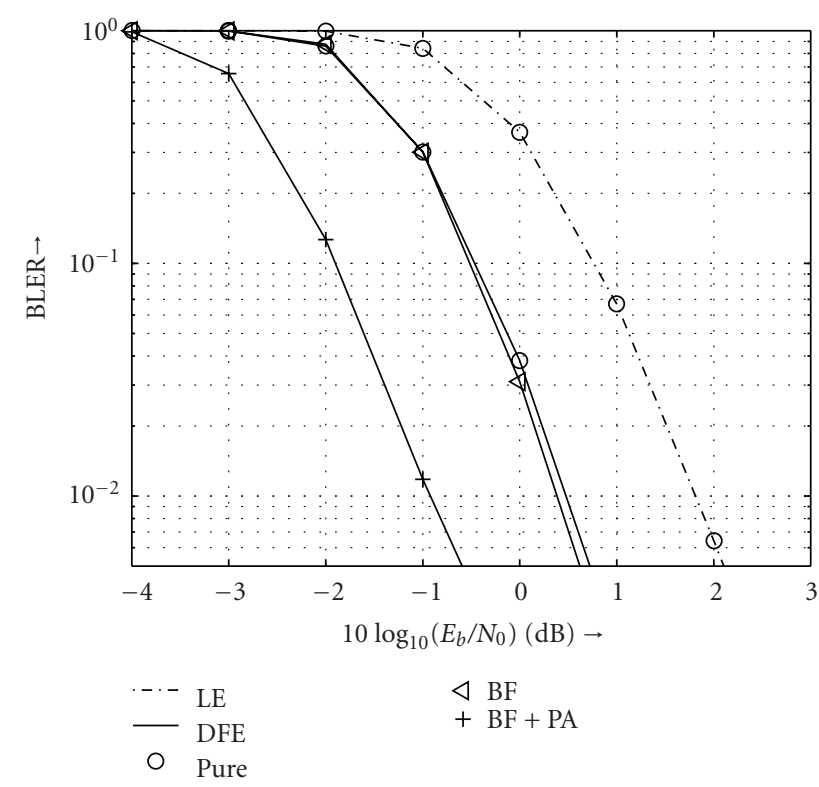

FIGURE 8: BLER for conventional MMSE-LE (dash-dotted line, “o”), conventional DFE (solid line, “o"), DFE with eigenbeamforming (solid line, “ $\triangleleft$ ”), and DFE with eigenbeamforming and MMSE power allocation (solid line, “+”). Exponentially decaying channel profile, $\sigma_{n}^{2}[\kappa] \sim \mathrm{e}^{-\kappa / 4}, \kappa \in\{0,1, \ldots, 20\}$.

the performance of DFE can be boosted significantly with the proposed MMSE power allocation. Compared to pure $\mathrm{LE}$, even a gain of more than $2 \mathrm{~dB}$ can be observed for DFE with MMSE power allocation.

For the results in Figure 8, the subchannels of the MIMO channel are given by an impulse response with order $q_{h}=$ 20 , where the corresponding variance of the taps $\sigma_{h}^{2}[\kappa]$ decays exponentially, $\sigma_{h}^{2}[\kappa] \sim \mathrm{e}^{-\kappa / 4}, \kappa \in\{0,1, \ldots, 20\}$. With this highly frequency-selective channel profile DFE with an MMSE power distribution yields again the best result, outperforming conventional DFE, DFE with eigenbeamforming (both show similar BLER), and LE.

A further analysis of the MSEs of the transmit streams after equalization (results not depicted) has shown that the application of eigenbeamforming leads from a balanced error level for both substreams in case of no beamforming to an unbalanced MSE pattern, where there is one strong substream and one weaker substream with unreliable symbols. Additional MMSE power distribution even tends to enlarge the difference between the substreams, which is preferable for a scheme with strong channel coding, as the reliable symbols can be exploited for error correction of less reliable symbols. On the other hand, if weak or no channel coding is applied, the weaker substream dominates the performance of the DFE. Hence, in this case MMSE beamforming leads to a higher BLER.

Finally, Figure 9 shows the complementary cumulative density function of the PAPR for SC-FDMA transmission, SC-FDMA with MMSE power distribution for DFE $\left(E_{b} / N_{0}=\right.$ $7 \mathrm{~dB}$ ) and different $N_{\theta}$, and orthogonal frequency-division multiple access (OFDMA) transmission. $N_{\text {set }}=2$ has been

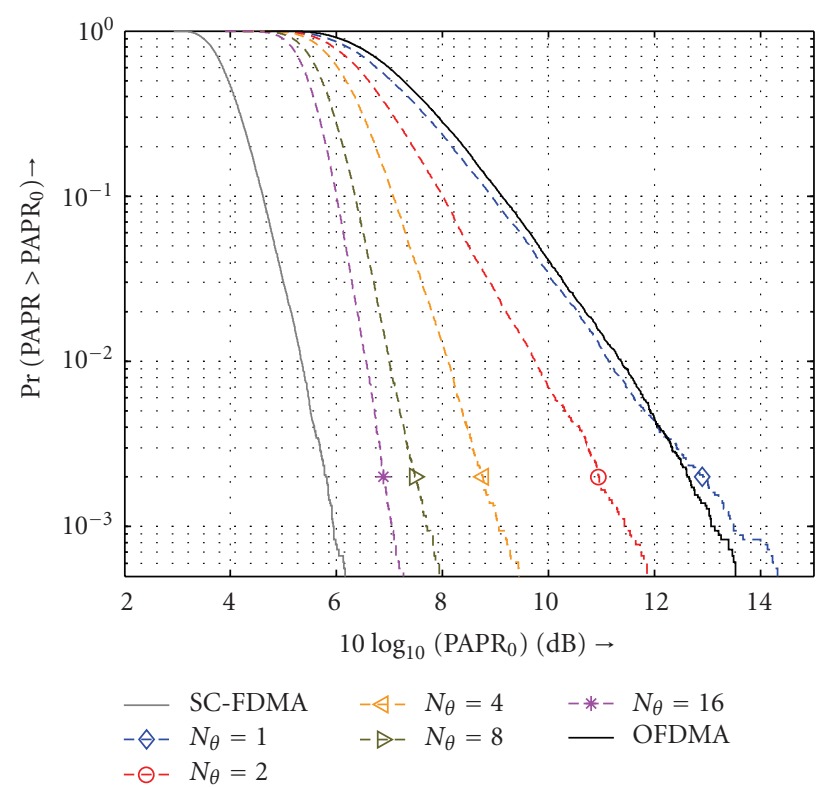

FIGURE 9: PAPR for SC-FDMA transmission without power allocation (solid gray), SC-FDMA transmission with beamforming and power allocation for DFE $\left(E_{b} / N_{0}=7 \mathrm{~dB}\right)$ (dash-dotted), and OFDMA transmission (solid black) for a Pedestrian $\mathrm{B}$ channel profile.

selected. Similar to LE, already $N_{\theta}=4$ is sufficient to reduce the PAPR significantly.

\section{Conclusion and Future Work}

In this paper, we have investigated the application of beamforming to spatial multiplexing MIMO systems with SCFDMA transmission. The transmitter was optimized for MMSE-LE and MMSE-DFE, respectively, at the receiver side. With the MMSE as optimality criterion, the derivations lead to an eigenbeamformer with nonuniform power allocation. Here, minimization of the arithmetic MSE for LE results in a power distribution scheme, where more power is assigned to poor frequencies which is in contrast to the classical capacity achieving waterfilling scheme resulting for minimization of the geometric MSE in case of DFE. This proves that eigenbeamforming with uniform power allocation, which was proposed in other work on beamforming for SCFDMA, is suboptimum. Simulation results confirmed these derivations. Because perfect feedback has to be assumed for a satisfactory performance of the beamforming scheme with DFE, the combination of Tomlinson-Harashima precoding and beamforming at the transmitter side should be investigated in more detail.

To mitigate the increase of the PAPR, which is caused by beamforming in general, the beamformer design was modified exploiting unused degrees of freedom without compromising optimality. Without affecting the BLER performance, rotations were introduced, where for transmission the combination of rotations with the lowest PAPR was chosen. It was shown that a small set of different angles of rotation 
is sufficient to obtain a significant PAPR reduction, hence, the additional computational complexity can be kept low. However, the angles of rotation used in the transmitter need to be communicated to the receiver for derotation after reception. Although a slight increase of the PAPR still remains, this seems to be acceptable considering the performance gain that can be achieved with beamforming and optimal power allocation.

In this work, we have assumed the availability of the exact beamforming matrices at the transmitter. In practice this is not possible, as it would cause a high overhead to feed back these matrices. Therefore, quantized beamforming matrices are fed back from the receiver to the transmitter in a real system, which reduces the transmission overhead but also leads to a channel mismatch. The impact of quantization on the performance remains to be determined in future work. Another open issue is to determine an optimization criterion that is more suitable for weak channel coding. It is known that for high code rates the weaker subchannel dominates the BLER performance. Therefore, a Min-Max optimization, where the maximum MSE of the substreams is minimized, seems to be a promising approach in this case.

\section{Acknowledgments}

This paper has been presented in part at the International ITG Workshop on Smart Antennas (WSA 2010), Bremen, Germany, February 2010, and has been presented in part at the IEEE Global Communications Conference (Globecom 2010), Miami, FL, December 2010. This work has been supported by Alcatel-Lucent Deutschland AG, Stuttgart, Germany.

\section{References}

[1] 3GPP TS 36.211, "Evolved Universal Terrestrial Radio Access (E-UTRA), Physical Channels and Modulation (Release 8)," 3GPP, June 2007.

[2] H. G. Myung, J. Lim, and D. J. Goodman, "Single carrier FDMA for uplink wireless transmission," IEEE Vehicular Technology Magazine, vol. 1, no. 3, pp. 30-38, 2006.

[3] T. Lunttila, J. Lindholm, K. Pajukoski, E. Tiirola, and A. Toskala, "EUTRAN uplink performance," in Proceedings of the International Symposium on Wireless Pervasive Computing (ISWPC '07), pp. 515-519, San Juan, Puerto Rico, January 2007.

[4] W. Gerstacker, P. Nickel, F. Obernosterer, U. Dang, P. Gunreben, and W. Koch, "Trellis-based receivers for SC-FDMA transmission over MIMO ISI channels," in Proceedings of IEEE International Conference on Communications (ICC '08), pp. 4526-4531, Beijing, China, May 2008.

[5] Y. Zhu and K. B. Letaief, "Single-carrier frequency-domain equalization with noise prediction for MIMO systems," IEEE Transactions on Communications, vol. 55, no. 5, pp. 10631076, 2007.

[6] D. Grieco, J.-L. Pan, R. Olesen, and N. Shah, "Uplink singleuser MIMO for 3GPP LTE," in Proceedings of the IEEE International Symposium on Personal, Indoor and Mobile Radio Communications (PIMRC '07), pp. 1-5, Athens, Greece, September 2007.
[7] H. G. Myung, J.-L. Olesen, and D. Grieco, "Peak power characteristics of single carrier FDMA MIMO precoding system," in IEEE Vehicular Technology Conference (VTC '07), Baltimore, Md, USA, 2007.

[8] T. Berger and D. Tufts, "Optimum pulse amplitude modulation, part I: transmitter-receiver design and bounds from information theory," IEEE Transactions on Information Theory, vol. IT-13, pp. 196-208, 1967.

[9] J. Yang and S. Roy, "Joint transmitter-receiver optimization for multi-input multi-output systems with decision feedback," IEEE Transactions on Information Theory, vol. 40, no. 5, pp. 1334-1347, 1994.

[10] R. W. Bäuml, R. F. H. Fischer, and J. B. Huber, "Reducing the peak-to-average power ratio of multicarrier modulation by selected mapping," Electronics Letters, vol. 32, no. 22, pp. 2056-2057, 1996.

[11] M. S. Back, M. J. Kim, Y. H. You, and H. K. Song, "Semiblind channel estimation and PAR reduction for MIMOOFDM system with multiple antennas," IEEE Transactions on Broadcasting, vol. 50, no. 4, pp. 414-424, 2004.

[12] S. Kay, "Some results in linear interpolation theory," IEEE Transactions on Acoustics, Speech, and Signal Processing, vol. 31, no. 3, pp. 746-749, 1983.

[13] B. Picinbono and J. M. Kerilis, "Some properties of prediction and interpolation errors," IEEE Transactions on Acoustics, Speech, and Signal Processing, vol. 36, no. 4, pp. 525-531, 1988.

[14] Y. Lebrun, C. Hofbauer, V. Ramon et al., "Tap and transmit antenna correlation based precoding for MIMO-OFDM systems," in Proceedings of the European Signal Processing Conference (EUSIPCO '09), Glasgow, Scotland, August 2009.

[15] S. Jafar, S. Vishwanath, and A. Goldsmith, "Channel capacity and beamforming for multiple transmit and receive antennas with covariance feedback," in Proceedings of IEEE International Conference on Communications (ICC '01), pp. 2266-2270, Helsinki, Finland, June 2001.

[16] M. Bazaraa, H. Sherali, and C. Shetty, Nonlinear Programming-Theory and Algorithms, Wiley-Interscience, Hoboken, NJ, USA, 3rd edition, 2006.

[17] D. Palomar, A unified framework for communications through MIMO channels, Ph.D. dissertation, Technical University of Catalonia (UPC), Barcelona, Spain, May 2003.

[18] J. Yang and S. Roy, "On joint transmitter and receiver optimization for multiple-input-multiple-output (MIMO) transmission systems," IEEE Transactions on Communications, vol. 42, no. 12, pp. 3221-3231, 1994.

[19] A. M. Chan and G. W. Wornell, "A class of block-iterative equalizers for intersymbol interference channels: Fixed channel results," IEEE Transactions on Communications, vol. 49, no. 11, pp. 1966-1976, 2001.

[20] Y. U. Zhu and K. B. Letaief, "Frequency domain preequalization with transmit precoding for MIMO broadcast wireless channels," IEEE Journal on Selected Areas in Communications, vol. 26, no. 2, pp. 389-400, 2008.

[21] M. V. Eyuboglu, "Detection of coded modulation signals on linear, severely distorted channels using decision-feedback noise prediction with interleaving," IEEE Transactions on Communications, vol. 36, no. 4, pp. 401-409, 1988. 